AERONAUTICAL QUARTERLY

A Journal devoted to Aeronautics and the Allied Sciences Volume XIX August 1968

Part 3

\title{
CONTENTS
}

A Calculation Method for Circulation Control by Tangential Blowing Around a Bluff Trailing Edge

R. J. Kind

The Shear Stiffness of Flat-Sided Corrugated Webs

A. Rothwell

Evaluation of the Separation Properties of Laminar Boundary Layers

C.Y. Liu

and $\boldsymbol{V}$. A. Sandborn

The Calculation of Heat Transfer Coefficients from Skin Friction Coefficients in the Compressible Laminar Boundary Layer on an Aerofoil

R. E. Luxton

Finite Plane Strain of Incompressible Elastic Solids by the Finite Element Method

J. T. Oden

A Unified Approach to the Initial Buckling of Stiffened Panels in Compression

W. H. Wittrick

The Cascade Subjected to Inlet Distortion

S. Fujii

LONDON

ROYAL AERONAUTICAL SOCIETY

4 HAMILTON PLACE, W1VOBQ 


\title{
THE AERONAUTICAL QUARTERLY
}

\author{
Editorial Board
}

Dr. A. M. BALlantyne, OBE, TD, BSc, PhD, FRAes, HonfCASI, FAiAA (Chairman)

Sir Robert CockBurn, KBE, CB, PhD, MSc, Fraes

Sir William Farren, CB, MBE, MA, DSc, FRS, Honfraes, HonfaiaA

Sir Arnold Hall, ma, frs, Honfraes, HonfaiaA

Professor D. W. HoldER, MA, FRS, FRAeS

Lord KINGS NORTON, DSc, DIC, PhD, FRAes, FAIAA

Sir Alfred Pugsley, obe, DSc, Frs, Honfraes

Sir GEOFFREY TAYLOR, MA, DSc, FRS, HonFRAeS

Dr. P. B. WALKER, CBE, MA, PhD, FRAeS

Editor-Miss E. C. Pike, MA, A praes

\section{Editorial Referees}

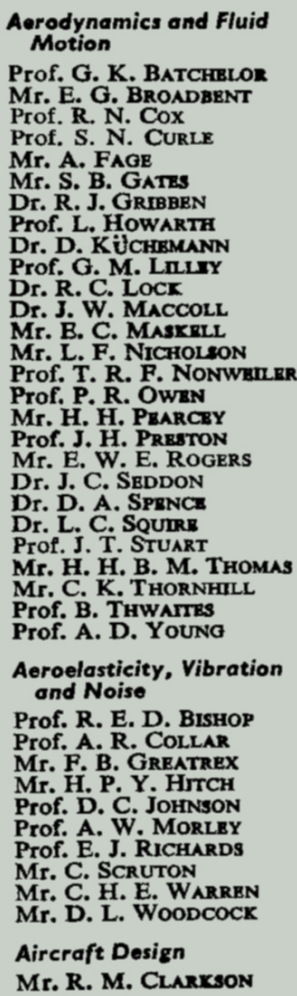

Dr. H. H. GARDNER

Mr. G. H. LEE

Mr. R. L. LICKLBY

Mr. M. B. MOROAN

Mr. F. H. POLlicutT

Dr. A. E. RuSSELL.

Dr. R. S. STAFFORD

Air Transport

Mr. A. C. CAMPBeLl ORDB

Mr. P. G. MASEFIELD

Mr. B. S. SHENSTONB

Mr. W. TYB

Astronautics and Guided

Flight

Mr. J. E. ALLEN

Mr. A. D. BAXTER

Mr. E. G. C. BUR'T

Mr. A. V. Clabaver

Mr. D. J. FARRAR

Mr. G. C. I. GARDINER

Mr. D. J. LYONS

Mr. W. H. STEPHBNS

\section{Aviation and Space}

Dr. K. G. Bergin

Dr. A. BUCHANAN BARBoUR

Dr. W. B. HICK

Control and Guidance

Mr. J. F. ATHERTON

Mr. B. G. GATBS

Dr. F. E. JONES

Mr. W. MAKINSON

Dr. R. L. SMTrH ROSE

Dr. A. M. UTTLEY

Instruments and Equipment

Dr. G. E. BAIRSTO

Mr. A. E. BINGHAM

Mr. W. J. CHARNLBY

Mr. R. GrahaM
Mr. C. D. Holland

Dr. C. S. HUDSON

Mr. C. N. JAQUes

Mr. H. TEMPLETON

Materials and Metallurgy

Prof. L. Artchison

Dr. N. P. Allen

Dr. W. M. DOYLE

Dr. G. FORREST

Sir NeviLl MotT

Dr. B. P. Muluns

Mr. L. ROTHERHAM

Dr. H. Surton

Dr. C. SYKES Mathematics and Numerical
Analysis

Dr. E. T. GoodwiN

Dr. S. H. Hollinadale

Prof. G. TEMPIB

Meteorology and Space

Environment

Mr. P. J. Mrade

Prof. R. S. SCORRR

Prof. P. A. ShepPard

Performance and Flight Testing

Mr. H. DAvIES

Mr. R. R. DUDDY

Mr. J. HANSON

Mr. P. A. HUrToN

Mr. D. R. NeWMAN

Propulsion

Mr. E. L. Bass

Mr. S. L. BRAGG

Mr. H. G. CONWAY

Dr. J. W. DRINKWATER

Sir A. H. ROY FEDDEN

Prof. W. E. GARNER

Dr. S. G. HOOKRR

Prof. J. H. HORLOCK
Mr. A. R. Howell

Mr. W. H. LINDSEY

Mr. P. LLOYD

Dr. E. S. MOULT

Mr. F. NDXON

Mr. F. M. OWNER

Mr. H. PEARSON

Mir. H. PEARson

Prof. A. G. SMTrH

Dr. B. S. STRATFORD

Rotorcraft and Air Cushion

Vehicles

Prof. J. A. J. BanNetr

Mr. R. HAFNRR

Dr. G. S. HisLop

Prof W. A. MAT

Mr. R. STANTON JONES

Structures and Solid Body

Mechanics

Prof. D. N. De G. Allen

Dr. I. M. Allison

Prof. J. H. ARGYRIS

Mr. R. J. ATKINSON

Dr. A. J. BARRBTT

Prof. A. H. Chilver

Mr. H. L. Cox

Mr. D. C. FRaNCls

Prof. R. Hill

Prof. H. G. Hopkins

Mr. H. B. HOWARD

Mr. E. D. KEEN

Dr. D. M. A. LegGetT

Dr. E. H. MANSPIELD

Mr. L. S. D. MORLEY

Mr. M. J. P. PARS

Prof. S. C. RgDSHaW

Mr. R. H. SANDIFER

Mr. R. L. WHEELER

Prof. W. H. WITrRICK

VTOL/STOL

Prof. D. Kerrth-Lucas

Dr. R. C. PANKHURST

Dr. JoHn WrLIAMS 